\title{
Análisis cualitativo del sistema educacional de la residencia en Ortopedia y Traumatología en tiempos del COVID-19
}

\author{
Máximo de Zavalía, Inés Pierro, Francisco Tálamo, José María Varaona \\ Servicio de Ortopedia y Traumatología, Hospital Alemán de Buenos Aires, Ciudad Autónoma de Buenos Aires, Argentina
}

\begin{abstract}
RESUMEN
La pandemia del COVID-19 afecta directamente la actividad de los residentes de Ortopedia y Traumatología. La gran carga horaria que requiere su formación está disminuida ampliamente en estos momentos. Suponemos que dicha capacitación se ve gravemente afectada principalmente en el aspecto práctico, y no tanto así en el teórico. Objetivo: Realizar un análisis cualitativo del sistema educacional de la residencia de Ortopedia y Traumatología en la Ciudad Autónoma de Buenos Aires y la región pampeana en tiempos del COVID-19. Materiales y Métodos: Se llevó a cabo un estudio de corte transversal que consta de una encuesta anónima de 16 preguntas de opción múltiple, orientadas a los cambios en la actividad teórica y práctica durante la pandemia. Se encuestó a 361 médicos residentes de Ortopedia y Traumatología que trabajan en la Ciudad Autónoma de Buenos Aires y la región pampeana. Resultados: Se recibieron 122 encuestas respondidas. El 76,2\% manifestó estar invirtiendo más tiempo en estudio que antes de la pandemia y el $71,3 \%$, no estar preocupado por su formación teórica. Distinto escenario se observa en la formación práctica, ya que el $88,5 \%$ refiere que su actividad quirúrgica disminuyó y el $90,1 \%$ manifiesta su preocupación ante tal situación. Conclusiones: Los residentes son conscientes del impacto que genera la cuarentena en su educación y muchos utilizan este período para fortalecer su formación teórica. Para enfrentar este desafío, son esenciales diversas estrategias, como telemedicina, webinars y videos sobre procedimientos quirúrgicos. Sin embargo, esta circunstancia sin precedentes nos deja como enseñanza lo complejo de suplantar la actividad práctica.
\end{abstract}

Palabras clave: Coronavirus; pandemia; educación médica; residencia; COVID-19.

Nivel de Evidencia: IV

Qualitative analysis of the Orthopedics and Traumatology residency system in times of COVID-19

\section{ABSTRACT}

COVID-19 pandemic has a direct impact on the daily practice of the Orthopedics and Traumatology residents. The considerable hour load demanded by their training is experiencing a severe reduction. We assume that their practical training is being seriously impaired, while their theoretical education has not been particularly hindered. Objective: To carry out a qualitative analysis of the Orthopedics and Traumatology residency system in the city of Buenos Aires and the Pampeana region during the COVID-19 pandemic. Materials and Methods: A cross-sectional study was carried using an anonymous survey of 16 multiple-choice questions oriented to changes perceived regarding the theoretical and practical training during the pandemic. The survey was distributed to 361 Orthopedics and Traumatology resident doctors who work in the city of Buenos Aires and the Pampeana region. Results: We received 122 answered surveys. Regarding their theoretical education, $76.2 \%$ of respondents stated that they were devoting more time to study than before the pandemic, and $71.3 \%$ that they were not worried about their theoretical education. The practical training perceptions present a different scenario as $88.5 \%$ of respondents stated that their surgical activities decreased and $90.1 \%$ expressed their concern regarding this situation. Conclusions: Residents are aware of the impact that the quarantine has on their training and many of them are using this period to improve their theoretical education. To rise to this challenge, relying on various strategies, such as telemedicine, webinars, and videos on surgical procedures, is essential. However, this unprecedented circumstance has made us aware of the major challenge involved in replacing practical training.

Key words: Coronavirus; pandemic; medical training; residency; COVID-19.

Level of Evidence: IV

Recibido el 17-5-2020. Aceptado luego de la evaluación el 19-6-2020 • Dr. MÁXIMO DE ZAVALÍA • maximodezavalia@gmail.com ID https://orcid.org/0000-0002-4022-4100 Cómo citar este artículo: de Zavalía M, Pierro I, Tálamo F, Varaona JM. Análisis cualitativo del sistema educacional de la residencia en Ortopedia y Traumatología en tiempos del COVID-19. Rev Asoc Argent Ortop Traumatol 2020;85(4):427-436. https://doi.org/10.15417/issn.1852-7434.2020.85.4.1120 


\section{INTRODUCCIÓN}

El aislamiento social, preventivo y obligatorio decretado por el gobierno argentino el 20 de marzo de 2020 tiene como fin disminuir la propagación del coronavirus o COVID-19. Esta resolución afecta directamente la actividad de los residentes de medicina en la República Argentina. Debido a dicha resolución, la gran carga horaria que conlleva la formación de los residentes se ve disminuida ampliamente.

Hasta el momento, 117 residencias médicas están acreditadas por la Asociación Argentina de Ortopedia y Traumatología (AAOT) y 31 están tramitando la acreditación. ${ }^{1}$ Cada una de ellas tiene diversos programas académicos y diferentes productos finales. ${ }^{2}$ A pesar de esto, la formación más completa de posgrado sigue siendo el sistema de residencias médicas.

La residencia de Ortopedia y Traumatología tiene dos pilares fundamentales: el aspecto práctico o quirúrgico y la actividad académica o teórica. Para la primera, la confianza, la competencia y, por sobre todo, el conocimiento de sus propias limitaciones hacen a una práctica quirúrgica más segura. ${ }^{3}$ En gran parte, esto puede perfeccionarse con "horas de quirófano" o bien en simuladores, ya sean de realidad virtual o físicos. En cuanto al aspecto teórico, suele fortalecerse con clases dictadas por los residentes o bien por los médicos de planta del hospital, ya sea a través de cursos de modalidad presencial, en línea o mediante la lectura adicional fuera del ámbito hospitalario. La gran carga horaria que tienen los residentes, principalmente en los primeros años de su capacitación, es inversamente proporcional al tiempo disponible para fortalecer sus conocimientos teóricos.

En muchas partes del mundo, se recurre al uso de la tecnología (teleconferencias, casos problema, realidad virtual, inteligencia artificial) para mantener la formación académica ${ }^{4,5}$ y planteamos como hipótesis que una situación similar está ocurriendo actualmente en nuestro entorno.

Nos planteamos como objetivo realizar un análisis cualitativo del sistema educacional de la residencia médica en la Ciudad Autónoma de Buenos Aires (CABA) y la región pampeana en tiempos del COVID-19.

\section{MATERIALES Y MÉTODOS}

Se llevó a cabo un estudio de corte transversal, cuyo universo corresponde a los residentes de la especialidad de Ortopedia y Traumatología de la CABA y la región pampeana (Entre Ríos, Santa Fe, Córdoba, Buenos Aires, La Pampa). Se realizó un cálculo del tamaño de la muestra mediante el empleo de una estimación de proporción considerando un número total de residentes de $428,{ }^{6}$ con un intervalo de confianza del $80 \%$, se tomó un tamaño de muestra de 119. El número total de residentes se estimó mediante la suma de la cantidad de vacantes obtenidas de la página web de la AAOT. Se confeccionó una base de datos tras contactarnos con un residente de cada institución. Se distribuyó una encuesta por correo electrónico, en la que se identificaba al grupo investigador y su propósito. Se solicitó completar dicha encuesta, que constaba de 16 preguntas con respuestas de opción múltiple (Anexo). Para recopilar la información demográfica del encuestado, se utilizaron las preguntas de la Encuesta sobre la Influencia COVID-19 en la Actividad de los Traumatólogos en Argentina, realizada por la AAOT. ${ }^{7}$ El resto de las preguntas hace hincapié en la forma de trabajo y el acceso a la actividad académica teórica y práctica durante la cuarentena. Se utilizó Google Formularios® para la confección y la distribución de la encuesta.

\section{RESULTADOS}

Se distribuyó la encuesta enviando un correo electrónico a 361 residentes. Se obtuvo una tasa de respuesta del $33,7 \%(\mathrm{n}=122)$. El 59,8\% de las encuestas proviene de profesionales de hospitales del ámbito privado y el 40,1\%, del ámbito público (Figura 1). Dentro del ámbito público, el 53\% corresponde a hospitales municipales; el 28,5\%, a hospitales provinciales y el 18,3\%, a nacionales. En cuanto a la distribución geográfica, la mayoría de los encuestados es de la CABA (77\%), mientras que el resto es de la región pampeana $(22,9 \%)$ (Tabla). La distribución jerárquica es la siguiente: $31,9 \%$ era R1; 26,2\%, R2; 21,3\%, R3; 15,5\%, R4 y 4,91\%, R5 o jefes de residentes (Figura 2).

El 87,7\% manifestó mantenerse en el mismo puesto de trabajo que antes del período de aislamiento, en tanto que un $9 \%$ ha sido trasladado a un sector de atención de pacientes con COVID-19. El 3,2\% no optó por ninguna de las opciones propuestas.

En cuanto a la asistencia al hospital, el 7,3\% seleccionó que asiste los 7 días de la semana; el 9,8\%, 6 días; el 10,6\%, 5 días; el 10,6\%, 4 días; el 12,2\%, 3 días; el 14,7\%, 2 días; el 27,8\%, un día y el 6,5\% no está concurriendo al hospital. Se interrogó acerca de la cantidad de guardias que estaban realizando por semana y el 36,8\% respondió una; el 29,5\%, dos; el 9,8\%, tres; el 4,9\%, cuatro; el 0,8\%, cinco; se dejó una opción para brindar una opinión para casos especiales, entre los cuales encontramos que el $10 \%$ concurre en grupos de trabajo (14 días cada grupo) y el $8 \%$ realiza guardias pasivas. 


\section{FORMACIÓN ACADÉMICA EN TIEMPOS DE COVID-19}

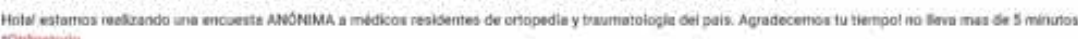
Oeslentevie

1 ¿Cial es tu ambito Hospitiario? Marca scio un Givaio

Pavise:

$\square$ puthican

2 Si el ambito es publico indique, (ai ho ellja no corresponde) *

Marea sclo is braio.

Muncipai.

Previsal

Nocianal

No comenponde

3 ¿Dentro de que region geopratica se cirtunscribe su Residencia?" Marca seito in braio

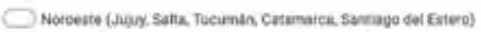

Nordente (Formosa, Oaco, Misiones, Cornemtes?

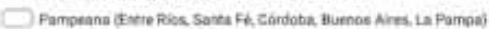

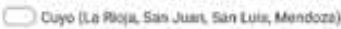

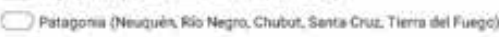

Caba

4. ¿ín que ìo de revidencla se encuentro actualmente?*

Marca sclo in brato.

$\bigcirc_{1}$

$\square_{2}$

$\square_{1}$

$\mathrm{S}_{4}$

$\square$

5. Soleccione la respuesta que mejor describa su sihuacicn en estos momensos do la pandemis. Marca scio in brato.

Me inantesgo en el miano poesto que anter del convento de la cinie del como.

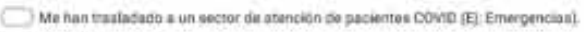

Drispens de etine

4. ¿Cuantos dias por semans concurre al Horpital? *

Marca scio un orato.

$D_{6}$

$\square$

$\square_{4}$

Q1

$\square_{2}$

$\bigcup_{1}$

No intor yosdo ai hosptas

7. ¿Cuahton guardias por semana? *

Marca solo un brato.

$\square^{\prime}$
$\square^{2}$
$\square^{2}$


8. En cuanto a la actividad acaserrica de tu resideneia. ¿ Estan haciendo clases o ateneos en TU HOSPITAL7. Mares solo in óraio.

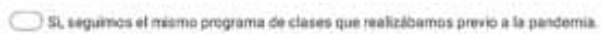

$\bigcirc$ si decimos adoptames y realuanot clates de smas de intere.

Na, la actovisad academica de mi resicencia se vio imterumpisa completamente por la pandemia.

9. ¿Utilizas el recirso de los Webinars o clases virtuales? *

Marca scle un braib.

$\square$ si

$\square_{\text {No }}$

Nolos canocis

10. En caso de utilizar los Webinan (si no los utliza, marcar No corresponde):

Marca solo un ovalo.

$\bigcirc$ solo veo los te la subespecialidad que me imeras.

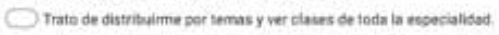

Veo allgurs que atro, no me quath al formato.

$\checkmark$ No coresponse.

11. En cuanto a tu formación teórica.

Marca solo en ovalo

Tespo mucho tiempo abre, ettoy aprovechansolo gara estuctiar y leer do ternas que antes no tenia tiempo.

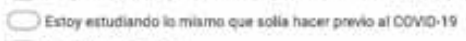

No estey estudiento

12. ¿Haz realizado o empezodo algün trabajo do imestigacion durante el periodo do Cuarentena? *

Marca solo un óvalo

Sis

No.

13. En cusnto a tu formación príctica:

Marca solo en ovalo

Sevis afectasta, solov vey a pocas chrogine.

Intento r a la mayot cantidad de cikugar poubiea.

14. ¿Te preccupa que tu formación teórica se vea afectada? *

Marca solo un óvalo.

$\bigcirc$ s

$\bigcirc$ No

15. 2Te preccupa que tu formacion practica se vea afectada?

Marce solo un ovalo.

$\bigcirc$

$\bigcirc$ no

16. ¿Sentis que el 2020 es un ahlo perdido en cuanto a tu tormacion? *

Marca solo en dovalo

$\square_{s}$

$\square_{\text {No }}$

Traiver 


\section{Ámbito hospitalario}

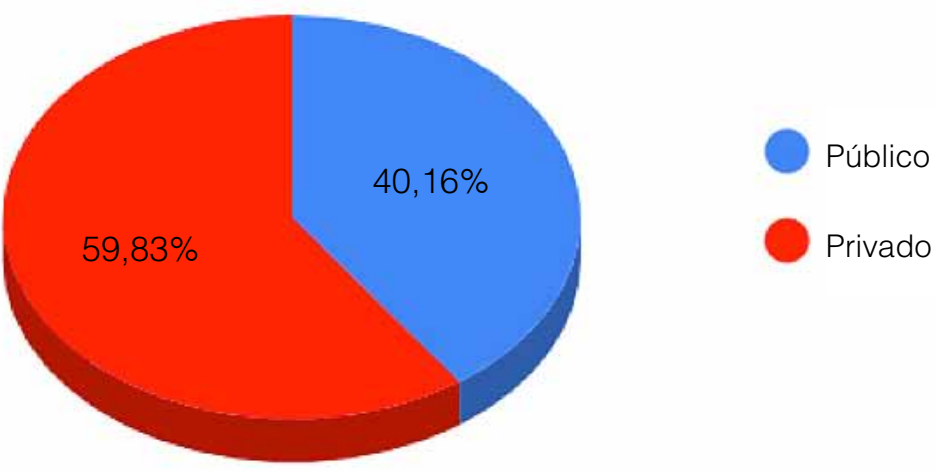

Figura 1. Distribución del ámbito de trabajo de los encuestados.

Tabla. Distribución demográfica de los encuestados

\begin{tabular}{|l|c|}
\hline Región & Cantidad de encuestados \\
\hline Ciudad Autónoma de Buenos Aires & 94 \\
\hline Región pampeana & 28 \\
\hline Total & 122 \\
\hline
\end{tabular}

\section{Año de Residencia}

100

75

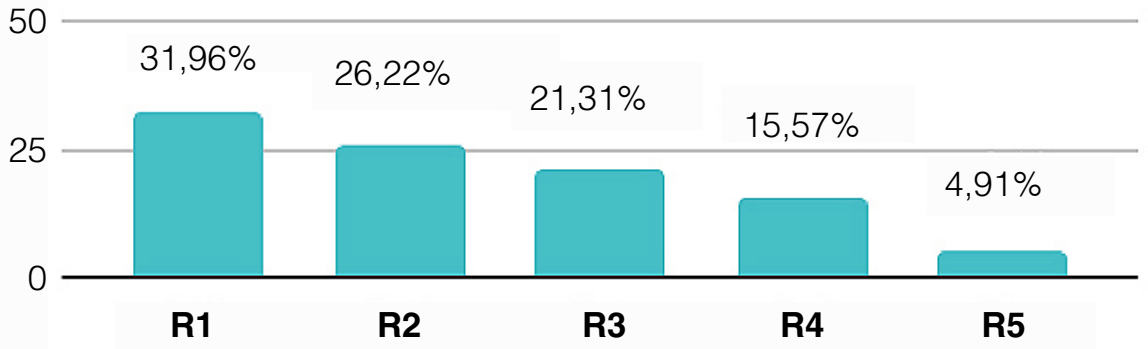

Figura 2. Distribución según el año de cursada de los encuestados. Se observa un mayor porcentaje de respuesta dentro de los primeros años. 
Respecto a la actividad académica propia de cada residencia, el $45 \%$ contestó que debió adaptarse al cambio y tomar clases según temas de interés, el $45 \%$ refirió seguir con el mismo programa académico anterior a la pandemia y el 9,8\% manifestó que la actividad académica se vio interrumpida completamente (Figura 3).

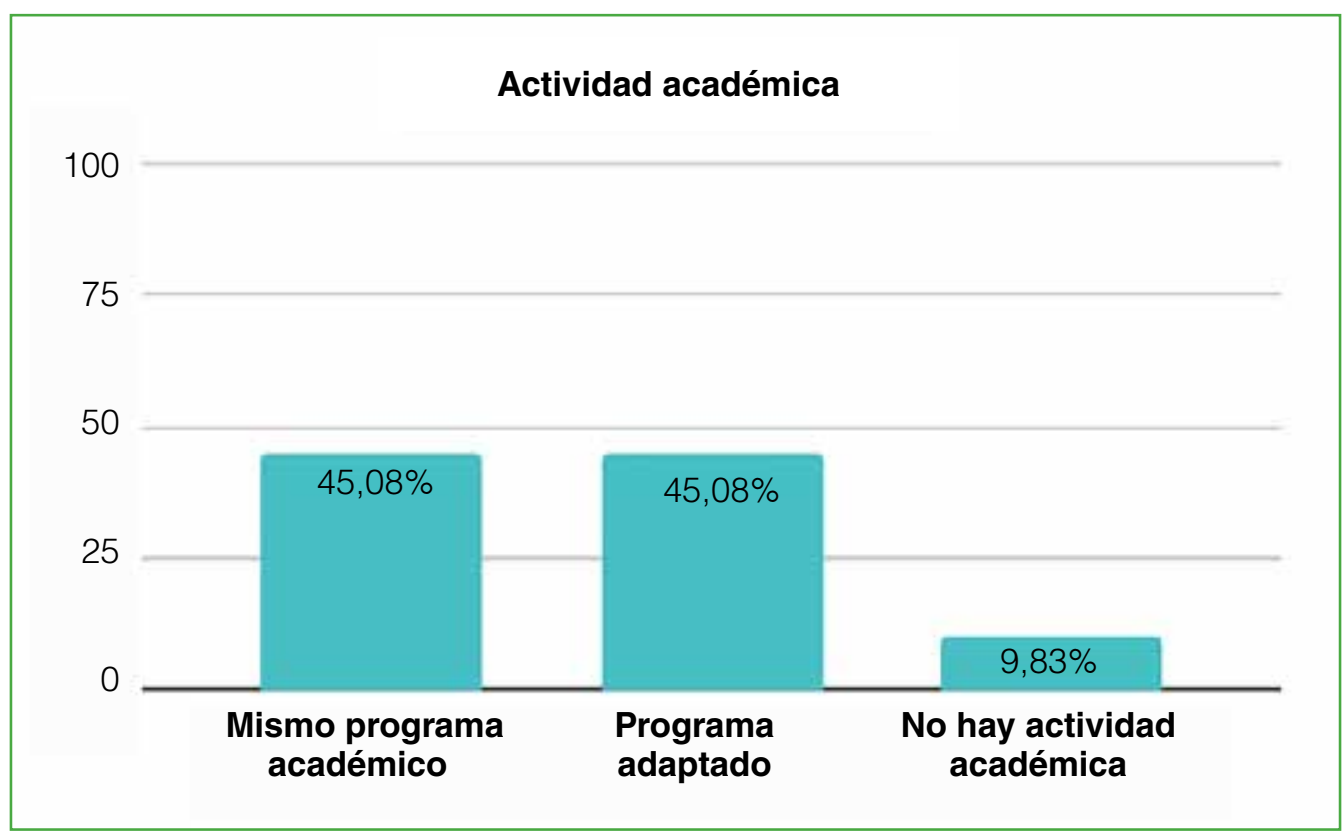

Figura 3. Tendencia de los encuestados con respecto a la actividad académica dentro de sus servicios.

La gran mayoría $(98,3 \%$ ) refirió tener conocimiento del recurso educativo de clases en línea o webinars, mientras que solo el 1,6\% contestó no conocerlo. En cuanto al uso de estos recursos, el $68 \%$ distribuyó su tiempo para leer temas generales de la especialidad, el 18,8\% solo mira clases de la subespecialidad que le interesa, el 7,3\% los utiliza poco debido a que no se siente cómodo con el formato y el 5,7\% no lo utilizan.

Al responder sobre la distribución del tiempo libre y su uso para la actividad teórica, el 76,2\% manifestó utilizarlo para estudiar y leer sobre temas a los que, antes de la pandemia, no les destinaba tiempo; el 20,4\% no refirió cambios en comparación con el tiempo dedicado al estudio y el 3,2\% dijo no estar estudiando.

El 61,5\% de los encuestados manifestó haber realizado o comenzado algún trabajo de investigación durante la pandemia (Figura 4). Por otra parte, en cuanto a la actividad práctica, la gran mayoría (88,5\%) dijo que se vio afectada, ya que solo concurre a pocas cirugías. 


\section{Actividad Científica}

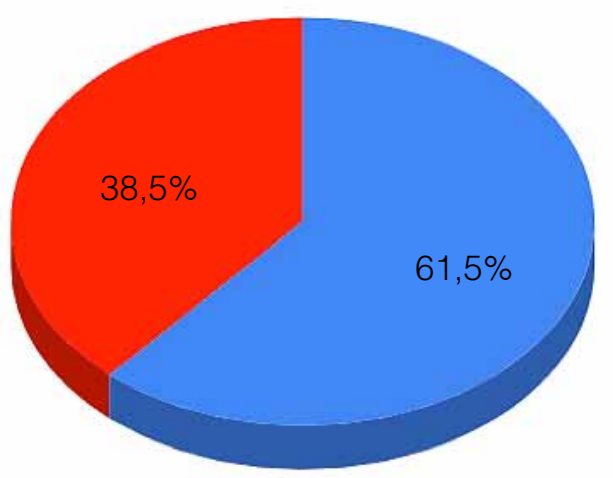

Comenzó o realizó un trabajo de investigación

No realizó un trabajo de investigación

Figura 4. Tendencia de los encuestados respecto de los estudios de investigación durante la cuarentena.

El 71,3\% informó no estar preocupado porque su formación teórica se vea afectada y un 28,6\% dijo estarlo. En cuanto a la formación práctica nos encontramos con resultados inversos, ya que el 90,1\% dijo estar preocupado y solo un $9,8 \%$ contestó que no lo está (Figura 5).

Por último, el 49,1\% respondió no sentir que el año académico actual es un año perdido, el 36,8\% contestó que tal vez y un $13,9 \%$ respondió que sí.

\section{Preocupación por la actividad práctica}

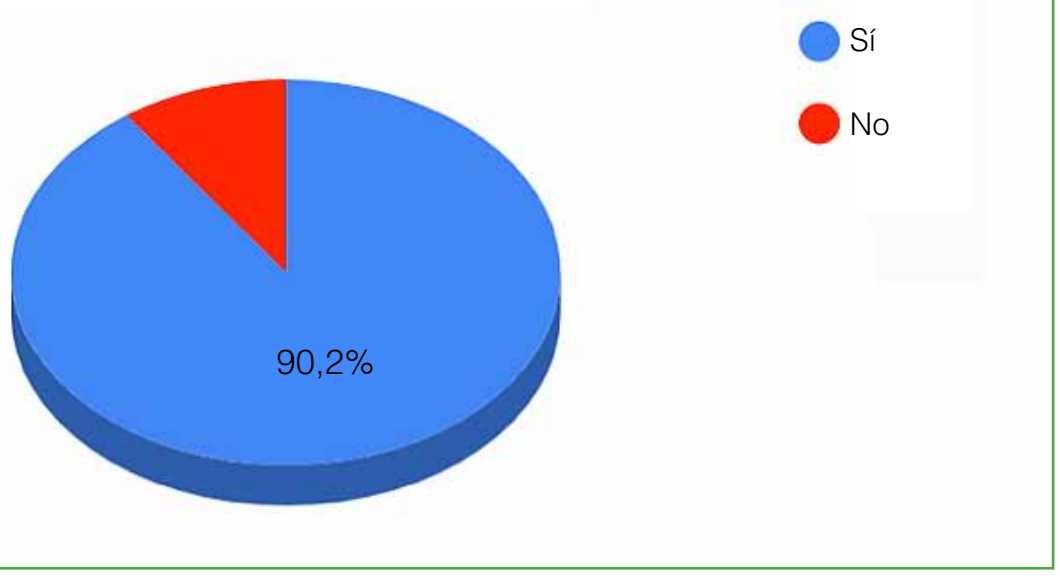

Figura 5. Preocupación de los encuestados por la falta de actividad y educación práctica dentro del marco de la pandemia. 


\section{DISCUSIÓN}

Es de público conocimiento que el residente médico cumple con un mínimo de concurrencia formativa de cinco días a la semana, más guardias y horas extra. Esto no sucede exclusivamente en la Argentina, también ocurre en países vecinos. ${ }^{8}$ Hoy en día, vivimos una situación especial, ya que los servicios han disminuido considerablemente la atención sanitaria y la actividad quirúrgica; ${ }^{9,10}$ en consecuencia, la concurrencia del personal médico, entiéndase también, de residentes médicos es en grupos de trabajo reducidos, lo que lleva a que las horas semanales de formación sean considerablemente menos. Tomando como universo la cantidad de vacantes disponibles de Ortopedia y Traumatología en la CABA y la región pampeana, nuestra muestra cumple con un intervalo de confianza del $80 \%$, por lo cual puede ser cuestionable su representatividad. Dicha muestra consiste en una mezcla heterogénea de residentes, representada por 49 hospitales públicos y 73 privados de los distintos años de la especialidad. En cuanto a la situación actual de formación de los residentes, pudimos observar que la gran mayoría $(87,7 \%)$ continúa realizando tareas formativas dentro de la especialidad sin haber sido trasladados a otro puesto donde debieran realizar funciones no relacionadas con la Ortopedia y Traumatología. Este dato se correlaciona con el estado actual de la curva epidemiológica del país, ya que, por el momento, no se evidencia un sistema de salud sobrecargado que haga necesario este tipo de cambios. Más de la mitad de los encuestados está concurriendo menos de tres días a la semana y, dentro de este grupo, un 6,5\% no está concurriendo al hospital. Los resultados en cuanto a la cantidad de guardias semanales que realizan son similares.

Cada programa de residencia se enfrenta a distintos desafíos según su ubicación geográfica e infraestructura. De acuerdo con los resultados, la disminución en la cantidad de horas de formación trae aparejado un impacto en la actividad académica, ya que el $45 \%$ debió adaptar sus cronogramas académicos y tomar clases según temas de interés. Por el otro lado, el $45 \%$ siguió su cronograma académico tal cual lo venía haciendo. Cabe resaltar que el $9,8 \%$ de los encuestados vio interrumpida completamente su formación teórica.

En la actualidad, contamos con recursos de clases virtuales o también llamados webinars, conocidos y utilizados por la gran mayoría de los encuestados $(98,3 \%)$, lo cual concuerda con la hipótesis planteada. Estos no son una novedad, porque, si bien son un recurso que existía antes de la pandemia, actualmente, han tomado un papel protagónico. Tienen como ventaja la posibilidad de acceder a clases gratuitas (en su gran mayoría) brindadas por especialistas y referentes de distintas regiones sobre diversos temas. Plataformas, como Zoom ${ }^{\circledR}$, Google Meet ${ }^{\circledR}$, YouTube ${ }^{\circledR}$, Facebook ${ }^{\circledR}$, Instagram ${ }^{\circledR}$, la página web de la AO, los múltiples cursos que ofrece la AAOT y sus asociaciones dependientes, le brindan al residente la oportunidad de continuar su formación teórica desde su hogar, conservando la calidad de la información.

Los residentes encuestados refieren disponer de más tiempo para realizar trabajos de investigación y la formación académica, esto resulta un aspecto muy positivo dentro de la negatividad que engloba la pandemia. Entendemos que una buena manera de aprender y formarse es realizando trabajos de investigación, ya que fomenta, en los autores, la lectura crítica de la bibliografía, y permite comprender el tema en cuestión, de una forma más detallada que la que puede aportar una sola fuente de información. Creemos que, durante este período, se debe hacer foco en convertir la amenaza en oportunidad mediante el estímulo de los formadores con el fin de promover y supervisar este tipo de actividades.

Otro escenario es el que ocurre con la formación práctica. La disminución del número de cirugías programadas afecta directamente la curva de aprendizaje quirúrgica del residente. Lo antes mencionado, se ve reflejado en la preocupación que expresa el 90,1\% respecto de su práctica quirúrgica. Sin duda alguna, este año deja muchos interrogantes. Consideramos que el más importante es determinar si 2020 es un año formativo perdido, dado que el 49,1\% expresó no sentir que estaba perdido y el 36,8\% dijo que tal vez. Esto nos hace suponer que, si bien el aspecto teórico ha sido enriquecido en estos últimos meses, el pilar práctico del residente no pudo ser sustituido en la misma medida, y generó, en los encuestados, dicho interrogante. Estos porcentajes representados en la encuesta realizada en mayo de 2020 son percepciones proyectivas de los residentes; por ende, no son porcentajes representativos, sino estimativos. Dicho interrogante debería ser planteado nuevamente al finalizar la emergencia sanitaria para llevar a cabo un nuevo análisis de manera retrospectiva.

Connor y cols. ${ }^{11}$ proponen diversas alternativas para la formación teórica de los residentes y nos hacen pensar que la situación actual nos dejará una enseñanza importante en cuanto a la formación teórica en los programas de las residencias médicas, esto coincide con los resultados obtenidos en nuestro estudio. Además, estos autores concluyen en que el pilar práctico tendrá un impacto significativo, porque no hay manera de remplazar las actividades prácticas presenciales, y proponen como alternativas para mitigar dicho impacto el uso de recursos, como videos quirúrgicos o realidad virtual. Otros autores describen similares accesos a la formación teórica comparados con los 
nuestros. ${ }^{12}$ En cuanto a la actividad práctica, cuentan con recursos de tecnología avanzada, como las estaciones de realidad virtual, aunque sostienen que su empleo no es igualable a la práctica quirúrgica. Proponemos como alternativa económica y aplicable a la situación actual del país el uso de bibliotecas virtuales que cuentan con videos de técnicas quirúrgicas de las distintas especialidades. Orthoflix Simall@ es un ejemplo de plataforma virtual utilizada por los médicos residentes en nuestra institución, cuenta con videos quirúrgicos dirigidos a médicos en etapa de formación, haciendo foco en la enseñanza virtual paso a paso de distintos procedimientos quirúrgicos.

Las limitaciones que enfrentamos al realizar el estudio fue la poca respuesta que obtuvimos en cuanto a la cantidad de hospitales contactados. Según una encuesta del Comité de Residentes y Sistemas Equivalentes de la AAOT, más del 70\% de la formación en dicha especialidad está en manos estatales, mientras que menos del 30\% corresponde a la asistencia privada. ${ }^{13}$ A pesar de esto, conseguimos más respuestas del ámbito privado que del público. No contar con una base de datos de correos electrónicos de residentes del país nos hizo limitar el estudio a la CABA y la región pampeana, ya que contamos con más llegada a dichos hospitales. Al evaluar la encuesta en retrospectiva, notamos que formular preguntas inductivas y ofrecer como respuestas las opciones binarias 'SÍ/NO' excluye un amplio espectro de respuestas posibles. Esto no nos permitió medir el grado de preocupación real de los residentes, como consecuencia de no dar lugar a la posibilidad de debate y las respuestas con matices. El planteo de este tipo de preguntas sesgadas e inductivas tuvo como resultado respuestas polarizadas y, principalmente, esperadas.

En conclusión, consideramos que los residentes son conscientes del impacto que tiene el aislamiento social, preventivo y obligatorio decretado por el gobierno argentino. Es evidente que los residentes están utilizando la cuarentena obligatoria para fortalecer su formación teórico-académica.

Nuestro estudio es el primero en proporcionar información sobre el posible impacto en el entrenamiento durante el actual período de emergencia sanitaria. Para enfrentar este desafío, son esenciales las diversas estrategias, como telemedicina, webinars y videos sobre procedimientos quirúrgicos. Sin embargo, esta circunstancia sin precedentes nos deja como enseñanza el déficit y lo complejo que es suplantar la actividad práctica. El análisis se llevó a cabo desde el inicio de la cuarentena hasta mayo inclusive, queda como interrogante las modificaciones que pueden ir ocurriendo a medida que transcurren los días. Creemos que este análisis de la situación realizado durante la pandemia puede servir para intervenir durante el problema y no una vez que concluya. A su vez, alentamos que se lleve a cabo un nuevo análisis una vez concluida la situación actual para poder realizar una evaluación final del daño o el impacto ocasionado.

Conflicto de intereses: Los autores no declaran conflictos de intereses.

ORCID de I. Pierro: https://orcid.org/0000-0002-6614-6921

ORCID de F. Tálamo: https://orcid. org/0000-0001-9060-6433

ORCID de J. M. Varaona: https://orcid.org/0000-0003-3540-4809

\section{BIBLIOGRAFÍA}

1. Listado de Residencias. Asociación Argentina de Ortopedia y Traumatología. Disponible en: https://aaot.org.ar/residencias/residencias-acreditadas. (Consulta: 8 de mayo, 2020)

2. Caviglia H. La educación médica en el ámbito de la Asociación Argentina de Ortopedia y Traumatología. Rev Asoc Argent Ortop Traumatol 2015;80(1):4-5. https://doi.org/10.15417/488

3. Borracci RA, Alvarez-Gallesio JM, Ciambrone G, Mezzadri NA. Autoestimación de habilidades y competencias quirúrgicas basadas en la teoría de la curva de aprendizaje en médicos residentes y becarios. Cir Cir 2019;87(4): 416-22. https://pesquisa.bvsalud.org/portal/resource/\%20es/mdl-31264986

4. Scullen T, Mathkour M, Maulucci CM, Dumont AS, Bui CJ, Keen JR. Impact of the COVID-19 pandemic on Neurosurgical Residency Training in New Orleans.World Neurosurg 2020;139:718-9.

https://doi.org/10.1016/j.wneu.2020.04.208 
5. Balakrishnan Ashokka, Say Yang Ong, Kwang Hui Tay, Ne Hooi Will Loh, Chen Fun Gee, Dujeepa D. Samarasekera. Coordinated responses of academic medical centres to pandemics: Sustaining medical education during COVID-19. Med Teach 2020;42(7):762-71. https://doi.org/10.1080/0142159X.2020.1757634.

6. Vacantes de residentes. Asociación Argentina de Ortopedia y Traumatología. Disponible en: https://docs.google.com/ spreadsheets/d/17XFGSn4kbagI2Nrt9BRp3jQn0giqHJ9S-FAjDCk_WJI/edit\#gid=0. (Consulta: 8 de mayo, 2020)

7. Encuesta sobre la Influencia COVID-19 en la Actividad de los Traumatólogos en Argentina. Encuesta de la Asociación Argentina de Ortopedia y Traumatología. Disponible en: https://es.surveymonkey.com/r/QYWYN7W. (Consulta: 8 de mayo, 2020)

8. Muñoz P N, Campaña W N, Campaña V G. Prevalencia del síndrome de burnout en los residentes de cirugía general de Chile y sus factores asociados. Rev Chil Cir 2018;70(6):544-50. http://dx.doi.org/10.4067/s0718-40262018000600544

9. Cattáneo A, Coppola EF, Federico Medina A, Pachón Guzmán A. Manejo y tratamiento de pacientes en áreas quirúrgicas durante la pandemia COVID-19 Revisión en el área de Ortopedia y Traumatología. Rev Asoc Argent Ortop Traumatol 2020;85(2):174-83. https://doi.org/10.15417/issn.1852-7434.2020.85.2.1101

10. Liebensteiner MC, Khosravi I, Hirschmann MT, Heuberer PR. Massive cutback in orthopaedic healthcare services due to the COVID-19 pandemic. Knee Surg Sports Traumatol Arthrosc 2020; Apr 30:1-7. https://doi.org/10.1007/s00167-020-06032-2

11. Connor Chick R, Clifton GT, Peace KM, Propper BW, Hale DF, et al. Using technology to maintain the education of residents during the COVID-19 pandemic. Brooke Army Medical Center, San Antonio, Texas; and University of California at San Francisco, San Francisco, California. J Surg Educ 2020;77(4):729-32. https://doi.org/10.1016/j.jsurg.2020.03.018

12. Schwarzkopf R, Maher NA, Slover JD, Strauss EJ, Bosco JA, Zuckerman JD. The response of an Orthopedic Department and Specialty Hospital at the epicenter of a pandemic: the NYU Langone Health Experience. $J$ Arthroplasty 2020;35(7S):S3-S5. https://doi.org/10.1016/j.arth.2020.04.041

13. Tello C, Vedoya S, Autorino C, Varaona J, Peláez R, Fazio A, et al. Encuesta dirigida a los Residentes sobre aspectos de la Residencia de Ortopedia y Traumatología. Rev Asoc Argent Ortop Traumatol 2017;82(2):150-6.

https://doi.org/10.15417/694 